\title{
El baile de los que sobran: cambio cultural y evaluación académica
}

\author{
El Baile de los que Sobran: Cultural Change \\ and Academic Evaluation
}

\author{
Yuri Jack Gómez-Morales* \\ Universidad Nacional de Colombia
}

\section{RESUMEN}

En este artículo de debate se examina el proceso de cambio cultural que está ocurriendo en las comunidades académicas y científicas colombianas, a partir del cual es posible entender las razones por las que, a pesar de lo desacertadas que puedan parecer las políticas de ciencia y tecnología $($ Cy $T$ ), particularmente sus modelos de medición (accountability) y sus rituales de validación (convocatorias, escalafonamientos, rankings, reclasificaciones, etc.), son adoptadas, apropiadas y operacionalizadas como consecuencia de la nueva cultura de la auditoría que se ha instaurado en nuestras instituciones universitarias.

Palabras clave: políticas de ciencia y tecnología, cultura de la auditoría, medición, cambio cultural.

\begin{abstract}
$\overline{\text { ABSTRACT }}$
This debate paper discusses the process of cultural change that is occurring in the Colombian academic and scientific communities, a change from which it is possible to understand the reasons why, despite their shortcomings, particularly their accountability models and validation rituals (calls, rankings, reclassifications, etc.), Science and Technology (Sci\&Tech) policies are adopted, appropriated, and operationalized as a result of a new audit culture that has been established in our university institutions.
\end{abstract}

Keywords: science and technology policies, audit culture, accountability, cultural change.

* Doctor en Sociología, University of York, Reino Unido. Profesor asociado del Departamento de Sociología de la Universidad Nacional de Colombia. Entre sus últimas publicaciones están: "Usos y abusos de la bibliometría" (2015), en Revista Colombiana de Antropología 51 (1): 291-307 y "Actitudes y valoraciones frente a la ciencia, la tecnología y la innovación" (2014), en Percepciones de las Ciencias y las Tecnologías en Colombia. yjgomezm@unal.edu.co. 
n primer lugar ${ }^{1}$, permítanme expresar mi agradecimiento a la organización del congreso ${ }^{2}$ y, en particular, al profesor Eduardo Restrepo, quien se tomó las molestias e hizo todas las gestiones para introducir este tema de la política de ciencia y tecnología en la programación del XVI Congreso de Antropología en Colombia. Y, por supuesto, agradecerle por su invitación a este espacio privilegiado de los debates centrales que me brinda la oportunidad de dialogar con la audiencia sobre este asunto de interés y preocupación pública que es compartida con Eduardo y, como se verá, con muchos de los asistentes a este evento, toda vez que se trata de un tema que está en el corazón de una especial manera de hacer política pública; me refiero a la medición de la ciencia, y más exactamente, la medición de la producción científica como forma privilegiada del new public management, de esta nueva forma de hacer política pública en $\mathrm{CyT}^{3}$.

La medición de la producción se constituye en forma privilegiada porque le permite a aquella política poner en marcha sus procesos de rendición pública de cuentas (accountability) y sus rituales de verificación, en torno de los cuales gira su credo de excelencia, transparencia, decisión informatizada, emprendimiento, competitividad. En fin, de un conjunto de valores que se originaron en el sector privado y comenzaron a implementarse como una nueva, mejor y más eficiente manera de administración de lo público, a comienzos de los años ochenta del siglo pasado en Inglaterra y Estados Unidos y, una década después, en América Latina, en lo que la antropología social denomina la cultura de la auditoría.

En efecto, a finales del siglo XX se produjo una impresionante proliferación del uso del término auditoría, que se extendió a contextos donde rara vez - o quizás nunca antes- había sido utilizado. La auditoría se convirtió en la palabra clave del vocabulario de la alta gerencia y fue el centro de gravedad para los hacedores de política y los Gobiernos de Occidente ${ }^{4}$. Esta cultura está configurada por

1 El baile de los que sobran da título a una canción de Los Prisioneros, grupo musical chileno de los años ochenta. La canción, escrita cuando Chile brindaba el primer escenario experimental para las reformas educativas neoliberales en América Latina, aborda el cierre de las oportunidades para la movilidad social de los jóvenes a través del trabajo y la educación.

2 Este artículo corresponde a la ponencia presentada en el debate central "Políticas de ciencia y tecnología en Colombia" en el marco del XVI Congreso de Antropología en Colombia y V Congreso de la Asociación Latinoamérica de Antropología, Pontificia Universidad Javeriana de Bogotá, el 8 de junio del 2017.

3 Este es un artículo de debate, categoría extinta y, por tanto, no reconocida por Colciencias ni practicada por su clientela.

4 Shore y Wright (2000, 58-59) comentan que la palabra auditoría etimológicamente se remonta al latín audire (oír, audiencia); y en sus múltiples definiciones evoca el principio de escrutinio, examen, superación de un juicio. En todas sus acepciones, la audiencia, el oír, tiene carácter 
prácticas que no se encuentran confinadas a ningún conjunto simple de instituciones ni a un lugar específico del mundo. Es una cultura reconocible en los más diversos lugares, ya que sus prácticas son muy aptas para gestionar también preocupaciones locales, como lo veremos más adelante. Estas prácticas determinan la asignación de recursos y resultan cruciales para la credibilidad de las organizaciones; sus miembros se tornan devotos de su implementación, pues evocan un lenguaje de aspiraciones comunes que los conecta entre sí y con el mundo (Strathern 2000, 3, 5, 9).

La cultura de la auditoría usa un viejo nombre para referirse a este fenómeno emergente: la rendición de cuentas generalizada. Su doble credencial como raciocinio moral y como método y precepto de la contabilidad financiera data de hace mucho tiempo. Un ejemplo de ello se encuentra en los trabajos de Babbage sobre seguros de vida de 1886 y su proyecto de mecanización del procesamiento de grandes series de números para facilitar el cálculo de las primas por parte de los actuarios. Pero, en el contexto de las prácticas administrativas, el ensayo inaugural de auditar las actividades de producción y distribución de la información se remonta al trabajo del economista Fritz Machlup, el primero en considerar el conocimiento (como información) en términos de recurso económico, abriéndole paso, así, a la noción de una sociedad de la información (Mattelart 2010, 43-45, 46-61). En The Production and Distribution of Knowledge in the United States, Machlup quería proporcionar un fundamento para la reforma de los sistemas de educación que los vinculara de manera más decidida al crecimiento económico, de allí que uno de los ejes del estudio fuese la medición de la productividad de estos sistemas de formación, razón por la que Machlup se inscribe dentro de la literatura bibliométrica de los “pioneros” (Gómez-Morales 2007). No obstante, de acuerdo con Mattelart, las incertidumbres sobre el crecimiento económico pronto socavarían las hipótesis del primer esquema de sociedad de la información, aunque su visión cientificista habría conseguido arraigar la idea de que las doctrinas organizacionales podían relegar lo político a un segundo plano y construir una sociedad gestionada según los principios de la dirección científica. A esta reorganización que implica una nueva función para el Estado como auditor y regulador, a esta tarea de racionalización se consagraría, entre 1960 y 1968, Robert McNamara, primer secretario de Defensa de Estados Unidos y, luego, presidente del Banco Mundial,

de inspección pública que asume un conjunto de "rituales de verificación" (Power 1997, 123) ante actores jerárquicamente superiores. En suma, la auditoría es una relación de poder entre quien examina y quien es examinado o auditado, viéndose este último en la obligación, que experimenta como responsabilidad, de rendir(se) cuentas traduciéndose a sí mismo en un sinnúmero de formularios y reportes, y asumiéndose como objeto de informaciones, pero nunca como sujeto en la comunicación; la auditoría no es comunicación (Foucault 1977, 200). 
cargo que desempeñó hasta 1981, esto es, hasta el inicio de la expansión del new public management. Este programa tuvo en los préstamos para el desarrollo, con sus términos, condiciones y matrices de evaluación, una herramienta imbatible en la propagación de la auditoría como práctica administrativa central en la configuración del Estado neoliberal (Harper 2000).

El fenómeno de la auditoría resulta tan cercano que a menudo se pasa por alto el juego de expectativas que conlleva y que florece en todo tipo de lugares de trabajo administrativo y profesional. Para muchos antropólogos, sociólogos y científicos sociales, la universidad resulta ser ese lugar de trabajo, y es justo allí donde la educación superior está siendo moldeada y administrada de acuerdo con lo que parece ser un consenso generalizado sobre sus fines, objetivos y procedimientos (Tratado de Bolonia ${ }^{5}$ ); un consenso que soporta y apoya la gobernanza a través de la doble exigencia de la eficiencia económica y las buenas prácticas académicas. Las prácticas en cuestión están relacionadas con la cotidianidad de la vida académica y, por tanto, tienen consecuencias directas y terriblemente nocivas, en opinión de algunos, para la producción intelectual. Con todo, como instrumento de rendición de cuentas que defiende las posibilidades de la internacionalización de la ciencia y la educación, la cultura de la auditoría resulta casi imposible de criticar, toda vez que promueve valores que los académicos, por lo general, aprecian mucho, como la responsabilidad, la libertad de investigación y la ampliación del acceso. Por eso, el reto de la cultura de la auditoría para la universidad es enorme, pues a través de ella lo financiero y lo moral se entrelazan en los preceptos de la eficiencia económica y la práctica ética.

Para orientar este debate, los organizadores del congreso comentaban en su invitación que, a pesar de las múltiples y diferentes voces que han cuestionado en los últimos años las políticas de ciencia y tecnología en Colombia, en particular aquellas concernientes a los cambios en los modelos de medición por sus criterios y las visiones de ciencia y de comunidad científica subyacentes, estas políticas son adoptadas y operacionalizadas. En este contexto, nos invitaron a elaborar una posible respuesta a la pregunta de por qué ha sucedido esto, así como a reflexionar sobre los impactos de estas políticas en las ciencias sociales y las posibles alternativas de acción frente a esta situación. Mi intervención en este debate se orienta fundamentalmente a ofrecer una explicación de las razones por las cuales estas políticas fueron aceptadas, adoptadas y operacionalizadas. 
La pregunta, sin embargo, es problemática en varios sentidos ${ }^{6}$; lo primero es que su formulación supone una suerte de alteridad radical entre un nosotrosque-es-un-yo, una comunidad científica y académica nacional, y un otro que formula, implementa y operacionaliza la política mediante los modelos de medición y otros mecanismos de ajuste institucional. No obstante, tal alteridad es una construcción ideológica que hemos elaborado para librarnos de la responsabilidad que nos corresponde como artífices y agentes de la política y sus medidas.

El segundo problema es que la pregunta implica o supone una cierta atemporalidad, como si este conflicto entre ese otro imaginado y este yo-que-es-unnosotros fuese constitutivo de estas dos identidades, como si fuese un conflicto que ha existido desde siempre, y eso tampoco es así. Mi impresión es que esta versión forma parte de la misma construcción ideológica que nos permite representarnos a nosotros mismos como un agente superior, cognitiva y moralmente, frente a ese otro imaginado, al cual se endilgan todo tipo de intencionalidades oscuras y macabras. Además, el conflicto tiene una temporalidad a partir de un conjunto de cambios que tuvieron lugar en el modelo de evaluación de Colciencias $^{7}$ (modelo de medición de grupos, 2012), que hasta entonces había permanecido con bajo perfil y por fuera de la esfera pública, como un asunto de expertos y políticos exclusivamente, que nos beneficiaba o nos resultaba indiferente, dependiendo de nuestra posición en el sistema universitario.

El tercer asunto, que no está enteramente explícito en la pregunta, es que el problema con el modelo de medición solo atañe a las ciencias sociales, las ciencias humanas y las humanidades. Claro que el modelo tiene consecuencias para estas comunidades, pero estas consecuencias traspasan las estrechas fronteras disciplinares que supone la pregunta, pues el modelo también tiene repercusiones del mismo tipo para las ciencias naturales, las ciencias exactas, las artes, el derecho y la economía: el distractor que se ha movilizado de una guerra de la ciencia a la criolla es solo un subterfugio. Y, así como las consecuencias atraviesan las fronteras disciplinares, atraviesan las fronteras geopolíticas, pues también en el norte global este modelo está produciendo subalternidades epistémicas del mismo tipo que se están produciendo en el sur global, como resultado

6 Textualmente, las dos preguntas orientadoras del debate propuestas por la organización del congreso fueron: "A pesar de las críticas planteadas, ¿por qué tales políticas terminan por ser apropiadas e instrumentalizadas?", y "pensando en el mediano y corto plazo, ¿cuáles podrían ser las acciones y los mecanismos más adecuados para enfrentar tales políticas?" (correo electrónico del 21 de mayo del 2017 de la organización del congreso para los invitados a debatir).

7 Departamento Administrativo de Ciencia, Tecnología e Innovación, entidad que formula y rige las políticas estatales de ciencia y tecnología en Colombia. 
de la implementación del new public management para la ciencia y la tecnología. De manera corolaria, entonces, debe entenderse que si bien nuestro debate de hoy parece muy parroquial, muy local, muy colombiano, este mismo debate está teniendo lugar en todo el mundo porque aquello a lo que nos estamos enfrentando es a la transición histórica, a escala global, de una ciencia modo 1 a una ciencia modo $2^{8}$, así como a la transformación y traslación concomitante de los lugares de producción de saber (Nowotny, Scott y Gibbons 2001). Parafraseando al viejo Nietzsche, lo que está en juego aquí también es el futuro de nuestras instituciones universitarias.

Pero, entonces, me dirán ustedes que cómo así que este debate que era sobre el Otro termina siendo un debate sobre Nosotros. Y sí, al menos en un componente muy central, la responsabilidad por estas políticas es nuestra. En mi opinión, antes del 2012, desde aquel momento constitucional (Jasanoff 2003) de finales de los sesenta del siglo pasado, cuando el proceso de institucionalización de la ciencia nacional formuló los mecanismos políticos de interlocución con el Estado en ese mítico seminario de Fusagasugá de 1968 (Ospina 1998), comenzó un paulatino proceso de transferencia, de delegación de la autonomía cognitiva - es decir, del poder-, pero, también, de la responsabilidad y el trabajo, que significa la validación social del conocimiento por parte de las propias comunidades científicas y académicas. Conforme fueron avanzando las décadas, fuimos delegando cada vez más autonomía en ese organismo que se convirtió en el “rector” de la ciencia y la tecnología, en ese leviatán llamado Colciencias; fuimos confiando más y más en sus permanentes y masivos procesos de evaluación con miras al "reconocimiento y escalafonamiento" de individuos, grupos, centros de investigación y revistas científicas. Esta delegación no fue el resultado de una enajenación o una expropiación, ¡fue voluntaria! Y es que, puesto en perspectiva, finalmente nosotros somos más positivistas de lo que quisiéramos creer y estaríamos dispuestos a aceptar. Esta delegación gradual fue el resultado de nuestra ingenua buena fe positivista, de nuestra oculta pero cómplice creencia en la ciencia, en que nosotros encarnamos esos valores modernos que son los que nos configuran como una élite social en el país, y nuestra certeza de que esos valores que ocultamente compartimos y nos constituyen como esa cosa indivisa a la que llaman la "comunidad científica nacional" eran también los valores sobre los que estaba fundado Colciencias. Si miramos los cuarenta años de su historia, no es difícil advertir la permanente presencia de representantes no elegidos de esta

Lo que ha provocado este escalamiento global de la transición es, justamente y entre otras
cosas, el proceso de estandarización y homogeneización que ha realizado el modelo de evaluación de la producción: la bibliometrización del mundo, parafraseando a Latour. 
élite que adquirieron posiciones en los cargos decisorios de aquella organización y tomaron decisiones cuya aceptación, en mayor o menor grado, estuvo en función del capital político que estos representantes pudieron movilizar en sus instituciones universitarias de origen. Silenciosa, casi secreta, esa confianza en la posibilidad de una política "científica" formaba parte de nuestra creencia, muy moderna, muy positiva, en la educación, la ciencia y la tecnología como motores del desarrollo. Este es el origen, en mi opinión, de un proceso de cambio cultural de las comunidades científicas y académicas.

Ahora bien, revisemos cuáles son las consecuencias de esta paulatina transformación cultural que se fue coproduciendo simultáneamente con el desarrollo de la nueva institucionalidad del sistema nacional de ciencia y tecnología. Para hacer breve la historia y enfocarnos en lo sustantivo, a mediados de la década de los noventa se comenzaron a aclimatar e introducir un conjunto de reformas estructurales en los sistemas de educación superior y de ciencia y tecnología. Por supuesto, estas reformas no surgieron ex nihilo, sino que venían amarradas, las primeras, a los compromisos del país, que ya tenía sus miras puestas en el ingreso a la Organización para la Cooperación y el Desarrollo Económicos (OCDE): por esta vía nos llegaron las pruebas Pisa ${ }^{9}$, así como todo el Bestiario de Bolonia ${ }^{10}$. Entretanto, nos hemos quedado mesmerizados con los rankings universitarios y con un modelo de educación superior que concibe este servicio como un mercado; y ahora, ya bien entrado el siglo XXI, comenzamos a entender que es un mercado global. Ya no se trata de la competencia entre UniAntioquia y UniNacional, sino entre universidades públicas y privadas y, próximamente, entre universidades nacionales y extranjeras, como resultado del aún incipiente pero inexorable proceso de internacionalización curricular que está comenzando a aclimatarse en nuestras instituciones universitarias. La otra gran agenda de política de ciencia y tecnología funcional para la educación es conocida como la agenda de internacionalización de la ciencia. Si nuestro sistema universitario estaba encaminándose al círculo de la excelencia, emulando los acuerdos de Bolonia para poder generar intercambios y flujos entre el norte y el sur, y mostrarle al mundo que al

9 Las pruebas Pisa (Programa para la Evaluación Internacional de Alumnos) miden los logros en matemáticas, ciencias y lectura de los estudiantes al final de la enseñanza obligatoria, entre los países de la OCDE.

10 El Bestiario es el producto de una iniciativa de estudiantes y profesores de la Universidad Ludwig-Maximilian de Múnich, que surgió en el verano del 2009, en medio de las movilizaciones de la comunidad académica en contra de la implementación de la reforma de Bolonia. Según sus autores, es una toma de posición crítica y política que se pregunta por la crisis de la universidad y su lenguaje mediante una parodia del Glosario de Bolonia, un compendio de los conceptos y expresiones que sustentan la reforma. Una selección del bestiario fue publicada en la Revista Literatura. Teoría, Historia, Crítica (2015) 17 (2): 227-242. 
menos en educación superior teníamos los estándares OCDE - lo cual supuestamente nos haría más competitivos en el mercado global-, entonces la investigación científica (que, dicho sea de paso, aún hoy sigue estando alojada de manera preponderante en las instituciones de educación superior) debería también internacionalizarse ${ }^{11}$. De esta manera, con los buenos oficios de la Comisión Nacional de Acreditación (CNA) del Ministerio de Educación y sus metodologías de autoevaluación con miras a la acreditación de alta calidad de instituciones universitarias y programas académicos, por un lado, y el diseño de una política de incentivos directos a la productividad internacional, el apoyo a redes, la configuración de grupos y el mejoramiento de las revistas nacionales, por otro, todos creímos - o una buena parte de nosotros lo hizo- que este proyecto de internacionalización de la educación, la ciencia y la tecnología era posible, bueno y necesario.

Ahora bien, estas reformas estructurales de los sistemas indujeron otro conjunto de reformas en las instituciones ejecutoras, una reforma en la cultura de la organización universitaria: oficinas de internacionalización en las universidades, movilidad académica, colaboración internacional, redes internacionales, grupos de investigación con miembros internacionales y publicaciones internacionales. En esto consiste la doble pinza de la reforma: el Ministerio-CNA con sus modelos de autoevaluación, y Colciencias y sus modelos de medición atrapan a las universidades que de buen grado hicieron el cambio en su cultura organizacional para convertirse en prisioneras de sí mismas, de los sistemas de evaluación y monitoreo que ellas ayudaron a construir y expandir, y como estas universidades están cautivas de estos sistemas, no tienen otra opción que presionar a sus asociados, los profesores universitarios, para cumplir con los objetivos y las metas que proponen los nuevos valores de la cultura de la auditoría. De allí que todo intento de insurrección termine sofocado desde adentro.

Así, en la práctica, las reformas estructurales a la educación superior introducidas por la agenda de aseguramiento de la calidad y la acreditación curricular e institucional de alta calidad, el summum de las buenas prácticas y la eficiencia financiera, operaron en todas las direcciones donde se creyó ver las deficiencias de la universidad pública, siendo una de las principales el exagerado poder que tenían sus docentes a partir del principio de la autonomía académica. La autonomía, para la cultura de la auditoría, o al menos para una vertiente Colombiano de Historia Social y de la Cultura 45 (19), en prensa. Mientras tanto, puede consultarse una versión oral en Foro Semana Mayo 2016: Mal Estado de la Ciencia Colombiana. https:// www.youtube.com/watch?v=FLWEbzJL7nk. 
de ella ${ }^{12}$, es vista como un obstáculo para la “empresarización” de la universidad y su integración funcional a las empresas que pretenden sus servicios. El poder de una universidad debe descentrarse de los docentes y entregarse a los administradores, entrenados en las buenas y necesarias prácticas para promover alianzas estratégicas y fecundas con los agentes privados, que son la nueva fuente de sostenibilidad financiera ante la retirada del Estado (De Sousa Santos 2007). Este summum de buenas prácticas y eficiencia financiera, esta racionalidad empresarial y mercantil socava la cultura universitaria al legitimar en su interior los discursos sobre calidad, competitividad y eficiencia en su evaluación y acreditación, en medio de políticas soterradas de fomento a las universidades privadas, de precarización de la situación de sus docentes con la reducción del presupuesto universitario y de difusión de la idea de que la educación superior es un "bien de consumo y no un derecho" (De Sousa Santos 2007).

Tal cascada de dispositivos, discursos, políticas e incentivos terminó por producir un nuevo cambio cultural en las comunidades académicas, consistente en modificaciones en los patrones de docencia, investigación y publicación que el modelo promueve. Por esta vía se generó una reconfiguración de la élite científica nacional, una élite que ya no está hecha de pares sino de administradores. En efecto, la nueva cultura de la auditoría se orienta fundamentalmente hacia el control del tiempo y, donde sea posible, a su aceleración. Volver todo módulos y cuantificarlo todo en ellos se vuelve la estrategia para reducir la duración de los estudios; controlar el tiempo de los estudiantes a través de cargas de trabajo se vuelve el objetivo de la docencia; incrementar la disponibilidad de los profesores es el objetivo de los jefes de unidades académicas (hoy llamadas centros de costo), y reglamentar una administración saturada, que cada vez se reparte más tareas, ponen en evidencia la preocupación por el tiempo y su aceleración que tiene la cultura de la auditoría. La otra cara de este trabajo sobre el tiempo se refiere a la presentación autopropagandística de las nuevas élites del conocimiento que buscan la gestión ante todo, pues todo tiene que funcionar perfectamente y estar disponible antes de la finalización del año académico; puede ocurrir que una decidida decana no le otorgue más de tres semanas a un instituto para desarrollar un programa de estudio, aunque los procesos administrativos tardan más de siete meses en su aprobación, porque todo debe estar consumado para su informe de gestión del final de año. Igualmente, puede suceder que una ministra de Educación henchida de emprenderismo convierta dos instituciones de educación superior en una sola, pero de “talla mundial” (traducción local del Bestiario 
de Bolonia), decisión de la que aquellas instituciones seguramente se enterarán por la prensa, como ocurrió con la notificación del cierre de las facultades de Ciencias Sociales, Humanas y Humanidades en Japón. Eso sí, la nueva élite se molesta muy poco por los procesos administrativos que, a causa del creciente incremento de la burocratización, hacen uso de lapsos cada vez más largos. Las élites en formación ven con malos ojos que se midan sus resultados: los otros son siempre los evaluados, acreditados, disciplinados y formateados. La auditoría es, esencialmente, una relación de poder entre quien realiza el escrutinio y quien es observado; esta se enfoca por entero en los objetos de información, nunca en los sujetos que se comunican (Ette 2015).

Ahora bien, la "nueva" clase trabajadora de la ciencia y la tecnología que encarna en sí los valores de la auditoría quedará relegada a cumplir el papel que la actual división del trabajo científico le impone a escala global, en un modelo que ha sido caracterizado como integración subordinada, basado en una práctica científica hipernormal que compromete las agendas nacionales de investigación, situación que resulta, en particular, patente en las ciencias médicas y naturales, pero que no se limita exclusivamente a estos campos ${ }^{13}$.

Quienes no se avengan a estos cambios terminaran "pateando piedras en el baile de los que sobran”, serán cada vez más invisibles, el sistema de oportunidades irá cerrándose paulatinamente para ellos y terminarán por desaparecer, junto con sus intereses, agendas y problemas de investigación, pues sus publicaciones ya no serán transables por reconocimiento, recursos y acceso a bolsas de financiación y beneficios. Lo triste, lo trágico de esta historia es, en palabras del filósofo Byung-Chul Han, que estos profesores subalternizados sentirán que fracasaron en la sociedad neoliberal del rendimiento y la productividad, se sentirán responsables por ello y terminarán avergonzados de sí mismos en lugar de poner en duda el sistema que los excluye de esta forma. En esto consiste el mecanismo de control de la accountability, de ese biopoder que redirecciona contra sí mismo la agresividad del individuo, anulando, de esta manera, al crítico, al revolucionario, al inconformista, y produciendo en su lugar un individuo depresivo y temeroso, que toma las decisiones y los cursos de acción, incluso cuando claramente los discierne como equivocados. Por esto es que, a pesar de lo equívoco de las políticas, estas siguen implementándose y operacionalizándose con la colaboración de todos nosotros.

Cfr. nota 3. Como medida paliativa para el espíritu de este nuevo tipo de trabajador habrá cursos de liderazgo, técnicas de mindfulness y todo tipo de prácticas de sí que lo salven de la anomia y que sofoquen el inconformismo y la protesta. 


\section{Referencias}

De Sousa Santos, Boaventura. 2007. La universidad en el siglo XXI. Para una reforma democrática y emancipatoria. La Paz: Cides-UMSA.

Ette, Ottmar. 2015. "Excelencia(s), velociferina(s). Sobre el bestiario de las élites engañosas de Bolonia”. Literatura: Teoría, Historia, Crítica 17 (2): 237-242.

Foucault, Michel. 1977. Discipline and Punish. Harmondsworth: Penguin.

Gómez-Morales, Yuri Jack. 2007. “Revisiting the 'Heroic’ Age: From Externalism to Internalism in Serial History of Science”. Ponencia presentada en la 11th International Conference of the International Society for Scientometrics and Informetrics, 25-27 de junio 2007, Madrid, España.

Harper, Richard. 2000. “The Social Organization of the IMF’s Mission Work: An Examination of International Auditing”. En Audit Culture. Anthropological Studies in Accountability, Ethics and the Academy, editado por Marilyn Strathern, 21-54. Londres: Routledge.

Jasanoff, Sheila. 2003. "In a Constitutional Moment: Science and Social Order at the Millennium”. En Social Studies of Science and Technology: Looking Back, Ahead, editado por Bernward Joerges y Helga Nowotny, 155-182. Dordrecht: Kluwer Academic Press.

Mattelart, Armand. 2010. Historia de la sociedad de la información. Barcelona: Paidós.

McDonald, Maryon. 2000. "Accountability, Anthropology and the European Commission”. En Audit Culture. Anthropological Studies in Accountability, Ethics and the Academy, editado por Marilyn Strathern, 106-132. Londres: Routledge.

Nowotny, Helga, Peter Scott y Michael Gibbons. 2001. Re-Thinking Science. Knowledge and the Public in an Age of Uncertainty. Londres: Polity Press.

Ospina, Martha. 1998. Colciencias 30 años. Memorias de un compromiso. Bogotá: Colciencias.

Power, Michael. 1997. The Audit Society: Rituals of Verification. Oxford: Oxford University Press.

Shore, Chris y Susan Wright. 2000. "Coercive Accountability: The Rise of Audit Culture in Higher Education”. En Audit Culture. Anthropological Studies in Accountability, Ethics and the Academy, editado por Marilyn Strathern, 57-89. Londres: Routledge.

Strathern, Marilyn. 2000. "Introduction: New Accountabilities”. En Audit Culture. Anthropological Studies in Accountability, Ethics and the Academy, 1-19. Londres: Routledge. 